\title{
How to Interpret the Pathological Report before and after Endoscopic Submucosal Dissection of Early Gastric Cancer
}

\author{
Dae Young Cheung and Soo-Heon Park \\ Department of Internal Medicine, College of Medicine, The Catholic University of Korea, Seoul, Korea
}

Possible lymph node metastasis (LNM) and residual cancer are major concerns in endoscopic submucosal dissection (ESD) for early gastric cancer. To reduce the risk of LNM and cancer recurrence, the proper indications for ESD should be considered. Histology, size, depth of invasion, and presence of ulceration should be thoroughly evaluated before proceeding with ESD. However, with incomplete information, discrepancies often arise between the pathological diagnosis based on the forceps biopsy and that based on the totally resected specimen. In addition, the presence of lymphovascular involvement and histological homogeneity can be clarified only after ESD. If the pathological diagnosis changes after ESD, we should reevaluate the curativeness and reformulate the goal of treatment. Additional surgery is a reasonable strategy for non-curative ESD, but a patient's other health conditions should also be considered. It is simple to read pathological reports before and after ESD, but it can be a complicated art to interpret the report and formulate an optimal approach. In this review, various considerations regarding the pathological diagnosis will be discussed. Clin Endosc 2016;49:327-331

Key Words: Endoscopic submucosal dissection; Early gastric cancer; Pathology

\section{INTRODUCTION}

The pathological diagnosis is made before and after endoscopic submucosal dissection (ESD) for early gastric cancer (EGC). The former is made from the forceps biopsy and the latter from the totally resected specimen. The interpretation of both pathological results is a key step in deciding the treatment strategy and predicting the outcome of ESD for EGC. Before performing ESD, we require information about the size, histological type and differentiation, and depth of invasion. However, there is often incomplete information and discrepancies can arise between the pathological diagnosis based on the forceps biopsy and that based on the totally resected

Received: June 21, 2016 Revised: July 5, 2016

Accepted: July 6, 2016

Correspondence: Soo-Heon Park

Department of Internal Medicine, Yeouido St. Mary’s Hospital, College of Medicine, The Catholic University of Korea, 10 63-ro, Yeongdeungpo-gu, Seoul 07345, Korea

Tel: +82-2-3779-1519, Fax: +82-2-3779-1331, E-mail: psheon5132@naver.com

(c) This is an Open Access article distributed under the terms of the Creative Commons Attribution Non-Commercial License (http://creativecommons.org/ licenses/by-nc/3.0) which permits unrestricted non-commercial use, distribution, and reproduction in any medium, provided the original work is properly cited. specimens. The forceps biopsy usually takes approximately 1.0 $\mathrm{mg}$ of tissue from the surface mucosa with each bite, and submucosal tissue is not included. Endoscopic ultrasonography (EUS) has value in regional lymph node evaluation but the accuracy for depth of cancer invasion is reportedly less than $50 \%{ }^{1}$ The choice of ESD or surgical gastrectomy depends on the pathological diagnosis obtained from the forceps biopsy specimen. After endoscopic resection (ER), we can examine the entire cancer specimen. We need to determine whether the pathological diagnosis is identical before and after ESD, whether the resection is complete and includes a safety margin, the degree of risk of lymph node metastasis (LNM), and whether there is need for additional surgery. In $1 / 3$ to $2 / 3$ of patients, the initial diagnosis changes after ESD. In addition, the presence of submucosal cancer cell invasion into lymphatics and vessels can be identified in the resected specimen. A mixed pattern of histology can also be evaluated in the totally resected specimen. These two findings also increase the possibility of LNM. If the pathological diagnosis changes after ER, we should reevaluate the curativeness and reformulate the goal of treatment.

In this review, various conditions relevant to the pathologi- 
cal diagnosis will be discussed.

\section{INTERPRETATION OF PRE-ESD PATHOLOGIC DIAGNOSIS: WHAT ARE THE PROPER INDICATIONS FOR ESD IN EGC?}

The indications should guarantee minimal risk of LNM and completeness of cancer removal. The factors that can affect these conditions are cancer size, depth of invasion, and histology. With advances in endoscopic techniques and instruments, the lateral size of cancer is no longer an obstacle to complete en bloc resection. Regarding the depth, submucosal invasion is a key component in predicting the possibility of LNM. The deeper the cancer invades into the submucosal layer, the higher the risk of LNM. Cancer with undifferentiated histology has greater risk of LNM and incomplete resection than cancer with differentiated histology.

What is the acceptable level of risk of LNM after ESD in EGC? We can infer the risk level by comparing the risk of surgical gastrectomy and ER. Surgical gastrectomy can remove the risk of metastatic lymph nodes but has the intrinsic risk of surgical complications, including mortality. If the risk of LNM after ESD is lower than the risk of mortality from surgical gastrectomy, we can reasonably consider ESD to be a better choice. Traditionally, mortality from surgical gastrectomy has been reported as around $1 \%$. A recent study comparing laparoscopic and open gastrectomy for EGC reported rates of $0.9 \%$ for mortality and $8.9 \%$ for significant surgery-related morbidity. ${ }^{2}$ Less than $1 \%$ risk is a reasonable threshold for recommendation of ESD over surgical gastrectomy.

Two levels are used in determining the indication for ESD. EGC that is confined to mucosa, spans less than $2.0 \mathrm{~cm}$, has no ulcer, and has differentiated histology defines an absolute indication (AI). The AI has been employed since the introduction of endoscopic mucosal resection, and has proven oncological safety and reliability, with an extremely low risk of LNM. The expanded indication (EI) includes EGC with differentiated histology, and nonulcerative mucosal cancer without size limitation, or ulcerative mucosal cancer less than $3.0 \mathrm{~cm}$, or non-ulcerative cancer $3.0 \mathrm{~cm}$ or less in diameter, with submucosal invasion less than $1 / 3$ of the submucosal layer, or less than $500 \mu \mathrm{m}$ from the mucosal muscle layer. The use of the EI was advocated by Gotoda et al., ${ }^{3}$ who reported that EGC cases that met the EI had no LNM on pathological review of surgically-resected specimens. A Korean retrospective study reported an acceptable oncological outcome for ER in EGC using EI. ${ }^{4,5} \mathrm{~A}$ total of 1,244 cases were analyzed. In a comparison of $\mathrm{AI}$ and $\mathrm{EI}$ groups, there were no significant differences in disease-specific recurrence-free rates. The local recurrence rate was $1.1 \%$ for the EI group and $0.9 \%$ for the $\mathrm{AI}$ group ( $p=0.783$ ).

However, with regard to AI and EI, we cannot confirm the characteristics of an EGC lesion before ESD. EGC with AI in pre-ESD evaluation can prove to be EGC with EI, or beyond expanded indication (BEI). Lee et al. ${ }^{6}$ reviewed 2,041 cases of gastric neoplasms and reported that $33.3 \%$ of EGC with AI turned out to be EGC with EI after resection. Moreover, 24.1\% of EGC with EI was finally diagnosed as EGC with BEI. Kang et al. ${ }^{7}$ also reported that one-fourth of EGC with EI before ESD proved to be EGC with BEI. The pre-ESD evaluation of EGC is not sufficiently reliable in terms of size, histology, differentiation, and depth of invasion. Therefore, even if EGC with EI can be treated safely with ESD, we should be cautious in using ESD for possible EGC with EI, and should prepare a follow-up plan for EGC with BEI.

EGC with undifferentiated histology is controversial. Gotoda et al. ${ }^{8}$ reported that 141 cases of intramucosal EGC less than $2.0 \mathrm{~cm}$ in size with undifferentiated histology showed no LNM in the surgical specimen. The pros for considering mucosal cancer less than $2.0 \mathrm{~cm}$ in size with undifferentiated histology as an indication for ESD are based on this report. Yoon et al. ${ }^{9}$ reported a $0.3 \%$ rate of LNM for mucosal cancer less than $2.0 \mathrm{~cm}$ in size with undifferentiated histology in Korean patients who underwent surgical gastrectomy. However, there are also cons. In a Korean study on 591 EGC cases with undifferentiated histology, $13.4 \%$ presented with LNM. Among those, mucosal cancer with LNM was identified in $2.9 \%$, which is higher than that seen in EGC with differentiated histology. ${ }^{10}$ Similarly, Lee et al. ${ }^{11}$ reported LNM in 3.2\% with mucosal cancer less than $2.0 \mathrm{~cm}$ in size with undifferentiated histology.

What accounts for the differences between studies on undifferentiated mucosal cancers? Two factors can affect the outcomes. First, the histological subtype of cancer with undifferentiated histology can matter. The other factor can be the size of the cancer. A size of $2.0 \mathrm{~cm}$ originates from the study by Gotoda et al., ${ }^{8}$ but we can reasonably adjust the cut-off size according to recent additional evidence. Park et al. ${ }^{12}$ reported different LNM rates according to the size of the undifferentiated mucosal cancer. The authors reported a 1.4\% (1/74) LNM rate for undifferentiated mucosal cancer less than $2.0 \mathrm{~cm}$, but if the cut-off moves to $1.5 \mathrm{~cm}$, LNM does not occur $(0 / 49){ }^{12}$ Chung et al. ${ }^{13}$ used cut-offs of $1.0 \mathrm{~cm}$ intervals. They reported a 1.6\% LNM rate for undifferentiated mucosal cancer less than $2.0 \mathrm{~cm}$, but 88 cases of mucosal cancer less than $1.0 \mathrm{~cm}$ showed no LNM. ${ }^{13}$ Kim et al. ${ }^{14}$ reported no LNM in mucosal cancer with poorly differentiated adenocarcinoma and signet ring cell carcinoma $1.5 \mathrm{~cm}$ or less in size. In summary, the 
LNM rate ranges from $0 \%$ to $3.4 \%$ for undifferentiated mucosal cancer less than $2.0 \mathrm{~cm}$ in size, but this value seems unsafe. However, if we narrow the cut-off size to 1.0 or $1.5 \mathrm{~cm}$ or less, we find no LNM cases, and meet the threshold level of $1 \%$ or less risk of LNM to advocate ER over surgical gastrectomy.

Undifferentiated histology comprises poorly differentiated adenocarcinoma, poorly cohesive cancer with a signet ring cell component, and mucinous carcinoma. Ye et al. ${ }^{10}$ analyzed the different LNM rates among the subtypes of undifferentiated histology EGC. EGC with poorly cohesive cancer and a signet ring cell component showed a significantly lower LNM rate of $6.0 \%$, compared to the $21.1 \%$ of poorly differentiated adenocarcinoma and $44.4 \%$ of mucinous cancer. Kim et al. ${ }^{14}$ also reported a lower LNM rate for EGC with poorly cohesive cancer and a signet ring cell component compared to that of EGC with poorly differentiated carcinoma (5.7\% vs. $14.2 \%, p<0.0001)$. Lee et al. ${ }^{15}$ reported a $0 \%$ LNM rate for both mucosal cancer $<2.0 \mathrm{~cm}$ with signet ring cell carcinoma, and mucosal cancer $<2.0 \mathrm{~cm}$ with well differentiated adenocarcinoma, in contrast to a $13.3 \%$ LNM rate for mucosal cancer $<2.0 \mathrm{~cm}$ with poorly differentiated adenocarcinoma $(p<0.001)$. A problem found in mucosal cancer with poorly cohesive cancer and a signet ring cell component is inaccuracy of measurement of the cancer size. There are two types of growth patterns in EGC with a signet ring cell component: expanding and infiltrative. Infiltrative cancer spreads along the deep layer of epithelium, and the overlying epithelial surface maintains a normal pattern. Endoscopic examination often fails to recognize the exact demarcation line of cancer. Except for this point, and based on the evidence above, we may consider mucosal cancer with poorly cohesive cancer and a signet ring cell component to be a proper indication for ESD. Of course, this may not be conclusive.

Regarding concerns about various levels, EGC with AI or EI can be treated safely with ESD, and the oncological outcome of ESD exceeds or is equivalent to that of surgical gastrectomy. ${ }^{7}$ Mucosal cancer with undifferentiated histology can be included in the indication, based on a size $<1.5 \mathrm{~cm}$ rather than $<2.0 \mathrm{~cm}$; among histological subtypes, signet ring cell cancer is more favorable.

\section{INTERPRETATION OF POST-ESD PATHOLOGIC DIAGNOSIS: IS THE TREATMENT FOR EGC COMPLETE?}

After ESD, we need to evaluate whether or not the treatment was curative. Curative resection with ESD in electrocardiography (ECG) is defined when cancer is resected completely, the resected specimen is histologically compatible with the indication, and the criteria are met for minimal risk of LNM. En bloc resection is important, and is a condition for curativeness. However, if the cancer was resected piecemeal and reconstructed intact, piecemeal resection can be regarded as curative. Additional histological information about curativeness that is available only after ESD is the presence of lymphatic or venous invasion. If we fail to achieve curative resection, we need an additional step to complete the treatment. Additional surgery is the most definitive strategy for failed ESD, but additional ESD or other ablative treatments can be considered when conditions permit. Close observation can be employed when additional treatment will carry more risk than benefit.

Resection margin involvement can be present as two types: lateral and vertical. When the lateral margin is positive, residual tumor is possible, but the risk of LNM does not increase. Excessive cauterization may narrow the lateral margin. Additional resection for the involved side of the ESD is proper.

Vertical margin positivity, lymphovascular (LV) invasion, and $>$ sm1 submucosal invasion are associated with an increased LNM rate. Oda et al. ${ }^{16}$ analyzed 226 cases of noncurative resection in which lateral margin positivity was excluded. After surgery, residual cancer was detected in $4.2 \%$ and LNM was positive in $6.3 \%{ }^{16}$

Lymphatic and vascular invasion are major histological predictors of LNM, but can only be determined after ESD. Most studies on the clinical significance of LV invasion are retrospective reviews of surgically treated EGC, and the outcome measurements vary in aspects and conditions. In analysis of undifferentiated EGC, lymphatic involvement by cancer cells increases the LNM rate from $6.7 \%$ to $62.0 \%(p<0.001) .{ }^{10}$ In studies on EGC with undifferentiated histology, the odds ratio for LNM ranges from 5.54 (95\% confidence interval [CI], 2.06 to 15.57 ) to 12.628 (95\% CI, 4.050 to 39.370$){ }^{10,12}$ For mucosal cancers, irrespective of differentiation, the presence of LV invasion increased LNM to $33.5 \%$ from $2.6 \%$ in LV-invasion-negative patients $(p=0.07) .{ }^{13}$ A study on mucosal cancer in poorly cohesive carcinoma with a signet ring cell component showed interesting results. The overall LNM rate was 3.7\%. ${ }^{17}$ However, if mucosal cancers less than $1.7 \mathrm{~cm}$ are counted, LNM was just 1.1\% (5/499) for LV-involvement-negative patients, and $33.3 \%$ (1/3) for LV-involvement-positive patients. For mucosal cancer with differentiated histology, LNM rate was not affected by the presence of $\mathrm{LN}$ involvement. ${ }^{18} \mathrm{~A}$ retrospective study on surgical specimens of non-curative ESD supports independence of LV involvement and LNM risk for mucosal cancer with differentiated histology. The LNM rate was $5.3 \%(6 / 113)$ in the LV-involvement-positive group and $6.2 \%(5 / 81)$ in the LV-involvement-negative group $(p=1.0){ }^{19}$ In summary, LV involvement is crucial for undifferentiated histology EGC, but possibly has no effect on differentiated 
histology mucosal cancer.

Should we consider mucosal cancer with undifferentiated histology to be noncurative? Due to incompleteness of a forceps biopsy specimen diagnosis, about 5\% of EGC with differentiated histology converts to undifferentiated histology after ESD. ${ }^{20}$ As noted above, if the undifferentiated cancer is confined to mucosa, measures less than 2.0 or $1.5 \mathrm{~cm}$, and has no LV involvement, we can use a follow-up strategy. Mixed histology is another concern. Evidence to date suggests that mixed histology cancer with undifferentiated predominance has highest LNM risk compared to other types, including pure undifferentiated histology EGC. ${ }^{21}$

Additional surgery for noncurative resection seems a reasonable strategy to avoid recurrence of residual gastric cancer. Kim et al. ${ }^{19}$ analyzed the long-term survival of patients with noncurative resection. Five-year overall survival rates in the additional surgery and untreated groups were $94.3 \%$ and $85 \%$, respectively ( $p=0.049$ ). However, a proportion of patients declined treatment, and other reports indicate the survival benefit from additional surgery is uncertain. Hoteya et al. ${ }^{18}$ analyzed survival of surgical and untreated groups. The 5-year survival rate was $96.9 \%$ for the surgical group and
$90.8 \%$ for the untreated group, but the difference was not significant $(p=0.089) .{ }^{18}$ Most deaths in the untreated group were from causes other than gastric cancer. Choi et al. ${ }^{22}$ reported that noncurative ESD cases occur in 7.1\% (89/1,246), due to deep submucosal invasion, and that among those, only $31.5 \%$ (28/89) underwent additional surgery. In this study, the overall survival in the surgery group was better than in the follow-up group ( $p=0.066$ ), but the disease-free survival of both groups was not different $(p=0.199)$. Most of the deaths were from pancreatic cancer, lung cancer, pneumonia, and cardiac arrest, rather than gastric cancer recurrence. This suggested that the non-surgical strategy was due to poor patient fitness for surgery.

\section{CONCLUSIONS}

With advanced technology and wide availability of endoscopic examination in Korea, over half of the gastric cancers present as EGC. ESD is less invasive, and preservative of organ function compared with surgical gastrectomy. However, pos-

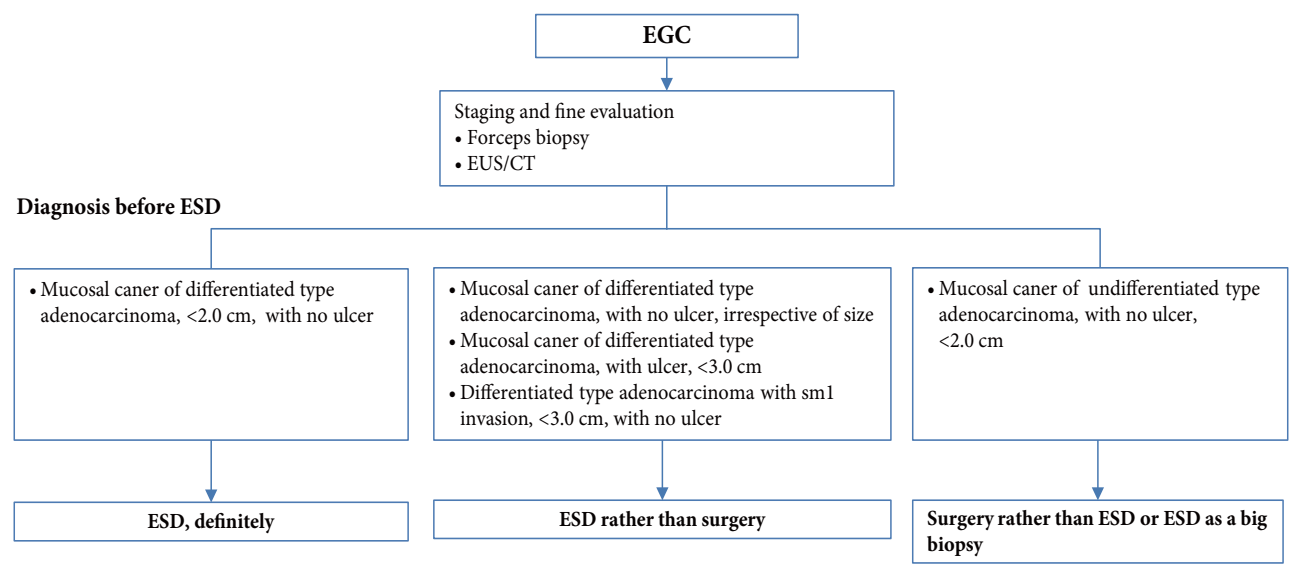

Diagnosis after ESD

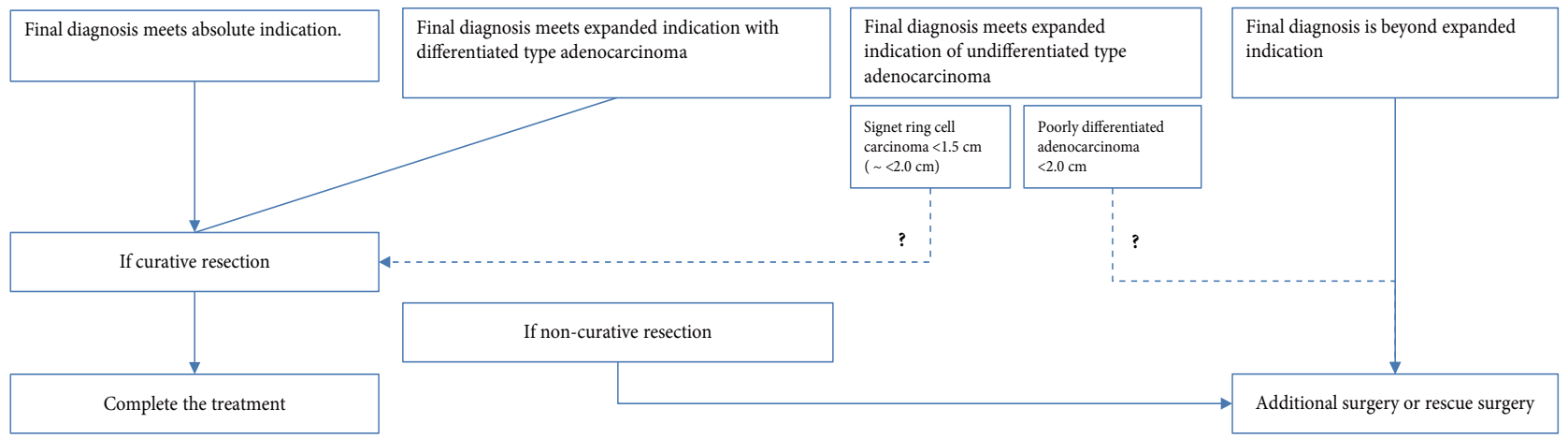

Fig. 1. Suggested algorithm for treatment of early gastric cancer (EGC) under various histological conditions. EUS, endoscopic ultrasonography; CT, computed tomography; ESD, endoscopic submucosal dissection. 
sible LNM and residual cancer after ESD are major concerns. To reduce these risks, proper indications for ESD in ECG must be determined. Along with careful endoscopic examination of the lesion, forceps biopsy and EUS are very helpful for increasing the accuracy of pre-ESD diagnosis. Histology, size, depth of invasion, and presence of ulceration should be thoroughly evaluated before proceeding to ESD. The presence of LV involvement and histological homogeneity are clarified after ESD. Additional surgery is a reasonable strategy for non-curative ESD, but a patient's other health conditions must be considered. It is simple to read pathological reports before and after ESD. However, it can be a complicated art to interpret the report and formulate an optimal approach (Fig. 1).

\section{Conflicts of Interest}

The authors have no financial conflicts of interest.

\section{REFERENCES}

1. Pei Q, Wang L, Pan J, Ling T, Lv Y, Zou X. Endoscopic ultrasonography for staging depth of invasion in early gastric cancer: a meta-analysis. J Gastroenterol Hepatol 2015;30:1566-1573.

2. Lee JH, Nam BH, Ryu KW, et al. Comparison of outcomes after laparoscopy-assisted and open total gastrectomy for early gastric cancer. Br J Surg 2015;102:1500-1505.

3. Gotoda T, Iwasaki M, Kusano C, Seewald S, Oda I. Endoscopic resection of early gastric cancer treated by guideline and expanded National Cancer Centre criteria. Br J Surg 2010;97:868-871.

4. Ahn JY, Jung HY. Long-term outcome of extended endoscopic submucosal dissection for early gastric cancer with differentiated histology. Clin Endosc 2013;46:463-466.

5. Ahn JY, Jung HY, Choi KD, et al. Endoscopic and oncologic outcomes after endoscopic resection for early gastric cancer: 1370 cases of absolute and extended indications. Gastrointest Endosc 2011;74:485-493.

6. Lee JH, Min YW, Lee H, et al. Diagnostic group classifications of gastric neoplasms by endoscopic resection criteria before and after treatment: real-world experience. Surg Endosc 2015 Dec 22 [Epub]. http://dx.doi. org/10.1007/s00464-015-4710-z.

7. Kang MS, Hong SJ, Kim DY, et al. Long-term outcome after endoscopic submucosal dissection for early gastric cancer: focusing on a group beyond the expanded indication. J Dig Dis 2015;16:7-13.

8. Gotoda T, Yanagisawa A, Sasako M, et al. Incidence of lymph node metastasis from early gastric cancer: estimation with a large number of cases at two large centers. Gastric Cancer 2000;3:219-225.

9. Yoon HJ, Kim YH, Kim JH, et al. Are new criteria for mixed histology necessary for endoscopic resection in early gastric cancer? Pathol Res Pract 2016;212:410-414.

10. Ye BD, Kim SG, Lee JY, et al. Predictive factors for lymph node metastasis and endoscopic treatment strategies for undifferentiated early gastric cancer. J Gastroenterol Hepatol 2008;23:46-50.

11. Lee JH, Choi MG, Min BH, et al. Predictive factors for lymph node metastasis in patients with poorly differentiated early gastric cancer. $\mathrm{Br} \mathrm{J}$ Surg 2012;99:1688-1692.

12. Park YD, Chung YJ, Chung HY, et al. Factors related to lymph node metastasis and the feasibility of endoscopic mucosal resection for treating poorly differentiated adenocarcinoma of the stomach. Endoscopy 2008; $40: 7-10$.

13. Chung JW, Jung HY, Choi KD, et al. Extended indication of endoscopic resection for mucosal early gastric cancer: analysis of a single center experience. J Gastroenterol Hepatol 2011;26:884-887.

14. Kim HM, Pak KH, Chung MJ, et al. Early gastric cancer of signet ring cell carcinoma is more amenable to endoscopic treatment than is early gastric cancer of poorly differentiated tubular adenocarcinoma in select tumor conditions. Surg Endosc 2011;25:3087-3093.

15. Lee SH, Jee SR, Kim JH, Seol SY. Intramucosal gastric cancer: the rate of lymph node metastasis in signet ring cell carcinoma is as low as that in well-differentiated adenocarcinoma. Eur J Gastroenterol Hepatol 2015;27:170-174

16. Oda I, Gotoda T, Sasako M, et al. Treatment strategy after non-curative endoscopic resection of early gastric cancer. Br J Surg 2008;95:14951500.

17. Pyo JH, Shin CM, Lee H, et al. A risk-prediction model based on lymph-node metastasis for incorporation into a treatment algorithm for signet ring cell-type intramucosal gastric cancer. Ann Surg 2016 Jan 7 [Epub]. http://dx.doi.org/10.1097/SLA.0000000000001602.

18. Hoteya S, Iizuka T, Kikuchi D, et al. Clinicopathological outcomes of patients with early gastric cancer after non-curative endoscopic submucosal dissection. Digestion 2016;93:53-58.

19. Kim ER, Lee H, Min BH, et al. Effect of rescue surgery after non-curative endoscopic resection of early gastric cancer. Br J Surg 2015;102:13941401.

20. Choi JM, Kim SG, Yang HJ, et al. Endoscopic predictors for undifferentiated histology in differentiated gastric neoplasms prior to endoscopic resection. Surg Endosc 2016;30:89-98.

21. Hanaoka N, Tanabe S, Mikami T, Okayasu I, Saigenji K. Mixed-histologic-type submucosal invasive gastric cancer as a risk factor for lymph node metastasis: feasibility of endoscopic submucosal dissection. Endoscopy 2009;41:427-432.

22. Choi JY, Jeon SW, Cho KB, et al. Non-curative endoscopic resection does not always lead to grave outcomes in submucosal invasive early gastric cancer. Surg Endosc 2015;29:1842-1849. 